\title{
La Formación de Profesores en Matemática a través de 40 años de la Revista Paradigma
}

\author{
Natalia Sgreccia \\ sgreccia@fceia.unr.edu.ar \\ https://orcid.org/0000-0003-2988-7410 \\ Facultad de Ciencias Exactas, Ingeniería y Agrimensura (FCEIA) \\ Universidad Nacional de Rosario (UNR) \\ Rosario, Argentina.
}

Recibido: 30/04/2020 Aceptado: 21/05/2020

\begin{abstract}
Resumen
Se realiza un recorrido por las publicaciones en la Revista a través de todos sus volúmenes y números, con especial atención a las relativas a la formación inicial de profesores en Matemática. A partir de la revisión efectuada, se identifican 12 artículos de interés, de autores iberoamericanos provenientes de seis países. Se reconocen dos principales asuntos centrales de tratamiento, relativos a perspectivas conceptuales relativas a la formación inicial de profesores en Matemática, con propuestas hacia planes institucionales, así como dispositivos específicos de formación inicial de profesores en Matemática, con especial énfasis en las TIC. Se comparte una síntesis del contenido de cada una de las obras ensayándose además relaciones conceptuales, a modo esquemático, entre las mismas. Finalmente, se comenta brevemente la línea de trabajo actual de la autora y su equipo de investigación en Argentina, que se encuentra en correspondencia con el tópico abordado.
\end{abstract}

Palabras clave: Formación inicial. Profesorado en Matemática. Planes de estudio. Dispositivos de formación.

\section{A Formação de Professores de Matemática através de 40 anos da Revista Paradigma}

\section{Resumo}

Uma leitura pelas publicações da Revista é realizado em todos os seus volumes e números, com atenção especial às relacionadas à formação inicial de professores de Matemática. A partir da revisão realizada, foram identificados 12 artigos de interesse, por autores iberoamericanos de seis países. São reconhecidas duas questões centrais de tratamento, relativas às perspectivas conceituais relacionadas à formação inicial de professores de Matemática, com propostas de planos institucionais, bem como dispositivos específicos para a formação inicial de professores de Matemática, com ênfase especial nas TIC. Uma síntese do conteúdo de cada uma das obras é compartilhada, e as relações conceituais são esquematicamente testadas entre elas. Por fim, é discutida brevemente a atual linha de trabalho da autora e de sua equipe de pesquisa na Argentina, que corresponde ao tópico abordado.

Palavras-chave: Formação inicial. Professores de Matemática. Planos de estudo. Dispositivos de formação. 


\title{
The Mathematic Teachers's Education through 40 years of Revista Paradigma ${ }^{1}$
}

\begin{abstract}
A reading of the published papers in the Journal related to pre-service training of teachers of Mathematics is made through all its volumes and numbers, with special attention to those related to the initial training of teachers in Mathematics. From the review carried out, 12 articles of interest were identified, by Ibero-American authors from six countries. Two main central issues of treatment are recognized, relative to conceptual perspectives related to initial teacher training in Mathematics, with proposals for institutional plans, as well as specific devices for initial teacher training in Mathematics, with special emphasis on ICT. A synthesis of the content of each of the works is shared, and conceptual relationships are schematically tested between them. Finally, the current line of work of the author and her research team in Argentina, which is in correspondence with the topic addressed, is briefly shared.
\end{abstract}

Keywords: Pre-service training. Career of Mathematics. Curriculum. Training's devices.

\section{Presentación}

Esta ocasión resulta un gran motivo para celebrar: cuatro décadas de publicación sistemática de la producción académica de calidad de investigaciones educativas en el contexto iberoamericano; particularmente con un espacio sostenido desde 1998 relativo a los trabajos desarrollados en la propia universidad donde radica la Revista ("La investigación en la UPEL"; con una primera aparición en 1982).

Fue una gran alegría haber sido invitada a esta celebración y mi primera tentación fue recorrer retrospectivamente la Revista, del último al primer artículo, como si fuera gradualmente retrocediendo en el tiempo.

Allí encontré un espectro ampliamente valioso de contribuciones y fui focalizando en las que están vinculadas con mi línea de trabajo actual: "la formación inicial de Profesores en Matemática".

En ese marco, he reconocido a 12 artículos que han tratado el tema en la Revista Paradigma. En lo que sigue procuraré compartir sus contribuciones, luego de haberlos agrupados según mi interpretación de los principales tópicos asumidos en el asunto de interés.

Este aporte constituye mi humilde regalo del 40 cumpleaños de Paradigma para el día 30 de julio del año 2020. Felicitaciones a la Universidad, al Equipo Editorial, a los autores, árbitros, lectores... Sostener estos espacios de encuentro de la comunidad de educadores iberoamericanos es altamente loable. ¡Y que vengan muchos años más!

\footnotetext{
${ }^{1}$ Revista periódica semestral, arbitrada, producida en el Centro de Investigaciones Educacionales Paradigma (CIEP) de la UPEL (Maracay, Venezuela). http://revistaparadigma.online/ojs/index.php/paradigma/index
} 


\section{Tópicos emergentes}

Los 12 artículos reconocidos relativos a la línea de investigación vinculada con la formación inicial de profesores en Matemática datan del año 1999 al año 2019 y fueron agrupados en dos tópicos, con igual cantidad de artículos cada uno, de acuerdo al principal asunto de tratamiento que asumen. Puntualmente se trata de perspectivas conceptuales relativas a la formación inicial de profesores en Matemática, con propuestas hacia planes institucionales, y de dispositivos específicos de formación inicial de profesores en Matemática, con especial énfasis en las TIC.

Estos dos temas entretejen las principales dimensiones por las que la línea investigativa se ha estado preocupando y ocupando: cómo conceptualizar y encuadrar en marcos teóricos lo relativo a roles, conocimientos, saberes e imaginarios en torno al profesor en Matemática y su formación esperada; de qué modo los planes de formación de las carreras para profesor en Matemática van amalgamando los cambios contextuales con las perspectivas teóricas, y van dando respuesta fundada a las necesidades sociales; en qué sentido acciones específicas del profesor en Matemática como la observación de clases, planificación de clases y vinculación de la disciplina con la realidad son potenciadas desde dispositivos orientados a ello; finalmente, y de manera puntual, de qué manera se integra la formación docente con la incorporación de las TIC para la promoción de actividades de enseñanza-aprendizajeevaluación de la Matemática que puedan emplearlas sustanciosamente.

\section{Perspectivas conceptuales relativas a la formación inicial de profesores en Matemática, con propuestas hacia planes institucionales}

En este agrupamiento se encuentran seis artículos que reflexionan sobre la importancia de revisitar el currículo de la formación inicial de profesores en Matemática en función a varios factores, tales como las necesidades contextuales en términos sociales, culturales, tecnológicos, económicos, las reformas educativas en los niveles educativos de desempeño, los avances en investigación educativa especializada así como la propia autoevaluación institucional. 
Los aportes provienen de colegas de Brasil, Chile, Colombia, Cuba, España y Venezuela, que desarrollan la línea investigativa en correspondencia con acciones de docencia y gestión en sus espacios de trabajo.

Es así que León Gómez (1999), de Venezuela, recomienda que los cambios que se dan en un cierto nivel educativo, como la educación básica, se correspondan con cambios en las carreras de Profesorado destinados a ese nivel.

En particular en la reforma venezolana de cara al siglo XXI se ideó un curriculum orientado hacia una enseñanza comprensiva, con centro en el estudiante, para propiciar el desarrollo de competencias que atiendan al ser, conocer, hacer y convivir.

Este enfoque propicia la interdisciplinariedad, particularmente desde ejes transversales de contenidos como son lenguaje, valores, desarrollo del pensamiento, trabajo y ambiente, para la formación de ciudadanos integrales, críticos y comprometidos con su entorno.

La autora comparte que, paralelamente, desde la UPEL se ha ejecutado un proceso de revisión curricular que considera a dicha reforma en el nivel básico de educación. La estructura general sigue contemplando cuatro componentes de formación: general, pedagógica, especializada y práctica profesional. En ellos se desarrollan asuntos relativos a los ejes transversales de la educación básica, pero de manera disjunta, con un enfoque solo teórico predominantemente y siguiendo en muchos casos el modelo tradicional de enseñanza.

Además, indica que cada docente desde sus acciones enseña a sus estudiantes una forma de ver la vida, de actuar y de pensar. Este accionar muchas veces tiene más peso sobre ellos que los conocimientos teóricos, científicos y didácticos. Es por ello que recomienda profundizar en la compaginación entre ser-hacer, acción-reflexión, teoría-práctica, trabajando a partir de situaciones específicas que se resuelvan con Matemática desde la transversalidad, desde las instancias de formación docente inicial y continua.

En esta línea González (2000), de Venezuela, comparte los principales retos que visualiza para la formación de profesores en Matemática de cara al siglo XXI. Comienza señalando el fenómeno mundial de la globalización y en ese contexto reconoce como uno de los desafíos primordiales lograr que los ciudadanos desarrollen capacidades robustas para tratar criteriosamente la abundante y dinámica información circulante por múltiples canales. Esto conlleva, a su vez, nuevos requerimientos matemáticos para los ciudadanos. 
Se trata de una alfabetización matemática que, más allá de conocimientos y razonamientos básicos, comprende también habilidades para comunicar matemáticamente a otros y emplear convenientemente las TIC para hacer Matemática. En este sentido, se trata de una Matemática que ayuda a las personas a comprender la realidad.

Una forma de lograrlo es ofreciéndoles problemas relevantes, que sean matemáticamente ricos y enriquecedores, abordables desde multiplicidad de disciplinas y vinculados con el entorno. El encargado de fomentarlo es el profesor en Matemática, para lo que está llamado a asumir nuevos roles en una escuela que crea nexos con personas e instituciones, si bien cercanas, externas a la misma (como padres, profesionales, clubes, empresas).

Se espera que el aprender Matemática sea parte de la autodisciplina de aprender para la vida, no solo para la escuela, con base en el trabajo colaborativo hacia metas compartidas así como el aprovechamiento del potencial de las tecnologías para el acceso y uso con sentido de la información. Acorde a ello, el profesor tiene él mismo desarrolladas estas habilidades y se constituye en investigador de su propia práctica. Se trata de un profesor que da oportunidades a sus estudiantes para comunicarse matemáticamente, para producir argumentaciones coherentes con base a información, para profundizar sus conocimientos matemáticos, para vincular la Matemática con otras áreas, entre ellas las humanísticas, así como con el entorno.

Es efecto, entre estas acciones se encuentra la labor esperada del profesor en Matemática que se está formando para desempeñarse laboralmente en el siglo XXI. Los planes de estudio de las carreras de grado quedan convocados a estar a la altura. Aquí radica uno de los retos identificados por el autor y no solo ello, los formadores de formadores también están llamados al cambio. Puntualmente convoca a realizar cambios en la formación inicial de profesores en Matemática a partir de los resultados de la investigación especializada y los llamados a realizar la transposición didáctica de tales resultados son los formadores de formadores.

Dado que la formación inicial de profesores en Matemática se caracteriza como un proceso de cambio conceptual y contextual, propone que los futuros profesores vivencien en primera persona las formas de hacer Matemática que se pretende que ellos luego lleven a cabo, mediante situaciones de enseñanza-aprendizaje-evaluación acordes. 
Un estudiante para profesor en Matemática puede repetir correctamente las definiciones que va estudiando durante la carrera, pero apelará a su propia imagen conceptual producto de su experiencia previa al momento de tomar decisiones que, por lo general, está teñida de obstáculos epistemológicos. De allí la relevancia de las experiencias con la Matemática que adquiera durante la carrera, ya que sus creencias se irán reforzando a partir de tales experiencias renovadas.

Acerca de las propias prácticas de enseñanza de la Matemática que va llevando a cabo el futuro profesor, estas no resultarán significativas si no se reflexiona sobre las mismas. Es en este sentido, y con base en una publicación previa, que el autor prioriza tareas intelectualmente exigentes en el período de formación. Sugiere, para ello, que las propuestas se sustenten en concepciones actuales de enseñanza-aprendizaje-evaluación de la Matemática y que en el aula de formación se produzcan situaciones análogas a las esperadas que el futuro profesor afronte. Todo ello con el fin de fortalecer el componente principal de los saberes de un profesor en Matemática: su conocimiento didáctico del contenido, constructo teórico propuesto por Lee S. Shulman (1986).

Un profesor en Matemática del tercer milenio prioriza interactuar con sus estudiantes a partir de interrogantes que promueven reflexión más que en el cúmulo de información. Requiere haberse apropiado de una visión histórico-social de la Matemática que le otorga relevancia a los procesos de la disciplina y le permita superar obstáculos epistemológicos. Como se viene insistiendo, lo que vivencie en el aula de formación estará alineado con lo que se espera de él.

También, resulta imperativo que un profesor en Matemática conozca él mismo las razones poderosas para estudiar Matemática y pueda ir asimilando los cambios contextuales en los que se desarrollan sus alumnos.

Por su parte, Pino-Fan y Godino (2015), de Chile y España, reconocen un creciente interés en el ámbito de investigadores en Educación Matemática por conocer más acerca de los conocimientos didácticos y matemáticos esperables en un profesor en Matemática para que enseñe de la manera más idónea posible. Al respecto, conviven distintos modelos teóricos que indican diferentes componentes a considerar como parte del conocimiento de un profesor en Matemática. Los autores consideran que se requieren análisis más detallados de los tipos de 
conocimiento puestos en juego por un profesor en Matemática, lo que redunda a su vez en fortalecer propuestas de formación inicial así como de evaluación de desempeño docente.

Se detienen en una versión ampliada, en términos de refinamientos y matizaciones, del modelo de conocimientos didáctico-matemáticos del profesor, bajo el enfoque ontosemiótico del conocimiento y la instrucción matemáticos. Este modelo contempla tres dimensiones: matemática (conocimientos común y ampliado del contenido), didáctica (facetas epistémica, cognitiva, afectiva, interaccional, mediacional y ecológica) y meta didáctico-matemática (idoneidad didáctica, normas y metanormas). Estas dimensiones se entretejen en las cuatro fases del proceso metodológico (estudio preliminar, diseño, implementación y evaluación) específicas de diseños instruccionales del tipo ingeniería didáctica.

Los autores indican que particularmente las seis facetas de la dimensión didáctica del conocimiento del profesor en Matemática sirven para comprender así como para promover a desarrollar dicho conocimiento del profesor, en tanto integración cognitiva de los conocimientos científico y práctico. Esto tanto desde las propuestas formativas de las carreras así como para ayudar a los profesores en Matemática a tener un nivel de conciencia explícito sobre los mismos. Señalan como una línea abierta de investigación al análisis de los criterios de idoneidad didáctica a objetos matemáticos concretos.

En particular, Parra-Sandoval y Villa-Ochoa (2017), de Venezuela y Colombia, indagan sobre la posibilidad de incluir una Matemática no institucionalizada en los modelos que describen el pensamiento de los profesores en Matemática, dado que han encontrado algunas limitaciones en los existentes. Se trata de una Matemática que no está presente en la cultura escolar pero sí en la cotidianidad. Se preguntan por la factibilidad de incorporación en el currículo escolar de esa Matemática y sus modos de proceder. También reconocen que en muchos de los intentos de llevar la realidad al aula de Matemática se trató de una realidad falseada o artificial.

A partir de varios ejemplos, observan que en la escuela muchas veces las decisiones matemáticas omiten factores extra-matemáticos que son importantes a tener en cuenta en el contexto real de la situación a resolver. Un primer punto pasa por valorar esos otros factores, consistentes en prácticas sociales no escolarizadas, otorgándoles valor tanto como a la Matemática institucionalizada. 
No ven posible adicionarlo como componente disjunto en los modelos existentes, dado que entienden el conocimiento del profesor como una conjunción de saberes, conocimientos, procedimientos y valores que se plasman en su hacer profesional diario. Sugieren prioritariamente discutir qué se entiende por Matemática y su aprendizaje, para desde allí continuar ahondando en el conocimiento profesional del profesor.

En este sentido, Fernández Peró, Hernández Murias, Sobrado Cárdenas y Sampedro Ruiz (2018), de Cuba, ofrecen fundamentos teórico-metodológicos para poner en diálogo a las disciplinas relativas a formación pedagógica general, Didáctica de la Matemática y formación laboral investigativa.

Puntualizan en las potencialidades del enfoque profesional pedagógico como alternativa para dicha integración. Sostienen que la integración entre las ciencias ha conllevado desafíos acordes a una nueva visión de la realidad dado que cambian los perímetros de las disciplinas. Esto también se asocia con la necesidad de experiencias que propicien una comprensión más reflexiva y crítica de la realidad.

De este modo sugieren que se disponga de orientaciones metodológicas para la planificación e implementación que estén dirigidas a los procesos de enseñanza-aprendizajeevaluación de saberes de manera integrada. Convocan a la investigación educativa a realizar aportes en este sentido.

Particularmente llaman a consolidar el núcleo integrador de formación laboral investigativa que tienen en la carrera, que propende a desarrollar habilidades profesionales que, al ser sistematizadas, brindan herramientas al futuro profesional para interactuar y transformar su objeto de trabajo a un nivel profesional.

Sucintamente se trata de la puesta en juego de conocimientos y habilidades de distintas asignaturas para idear soluciones interdisciplinarias a actividades vinculadas con la práctica laboral mediante la búsqueda, indagación, investigación y descubrimiento, con el uso de TIC.

En efecto, sugieren propiciar acciones orientadas a acercar el aula universitaria y la escuela, comunicarse con el entorno profesional, buscar respuestas científicas a los problemas de actuación profesional, concebir a lo profesional como elemento integrador en la carrera, sistematizar conocimientos y habilidades que se han integrado al dar solución a los problemas profesionales, propiciar la investigación educativa para abordar la realidad, desarrollar interés 
por la profesión, interrogar los contenidos, fomentar autoevaluaciones para reconocer errores con sus posibles causas didácticas.

Es en este marco que proponen tareas para la comprensión y dominio del nuevo contenido, para la sistematización así como para la aplicación del contenido. Reconocen a la práctica como una fuente importante de conocimiento para una teoría más enriquecida y también recíprocamente a la teoría para enriquecer la práctica, su aplicación y generalización.

Al respecto, Gomes de Oliveira y da Silva (2019), de Brasil, se preguntan cuál es el profesor en Matemática deseable constituido por el discurso de la Educación Matemática Crítica. También cuestionan cómo esta idea se plasma en la propia identidad del curriculum, producido social e históricamente, en tanto operación de poder.

De acuerdo a esta perspectiva, la Matemática se constituye en un medio mediante el cual el profesor ayuda al estudiante a ser un sujeto más consciente, crítico y humano, con capacidad para participar en la democratización de la sociedad, con autonomía intelectual y personal.

Esto incumbe a un profesor en Matemática que repiensa su práctica docente desde la perspectiva de los estudiantes. En efecto, construye un proceso de comunicación centrado en ellos, los escucha y minimiza relaciones arbitrarias de poder que pudieran inhibirlos.

Resumidamente, cuando un profesor en Matemática se ha ido configurando de acuerdo al discurso del currículo crítico, repiensa los contenidos matemáticos desde una postura de enseñanza crítica, que problematiza cuestiones sociales y políticas involucrando la Matemática.

A continuación se comparten tres esquemas (Fig. 1 a 3) que procuran sintetizar las contribuciones en este primer grupo de artículos.

En la Figura 1 se comparten los apuntes relativos a la necesidad de articulación de cambios en los distintos niveles implicados en las reformas curriculares. 
Figura 1. Articulación de cambios entre los diversos estratos implicados en los cambios educativos

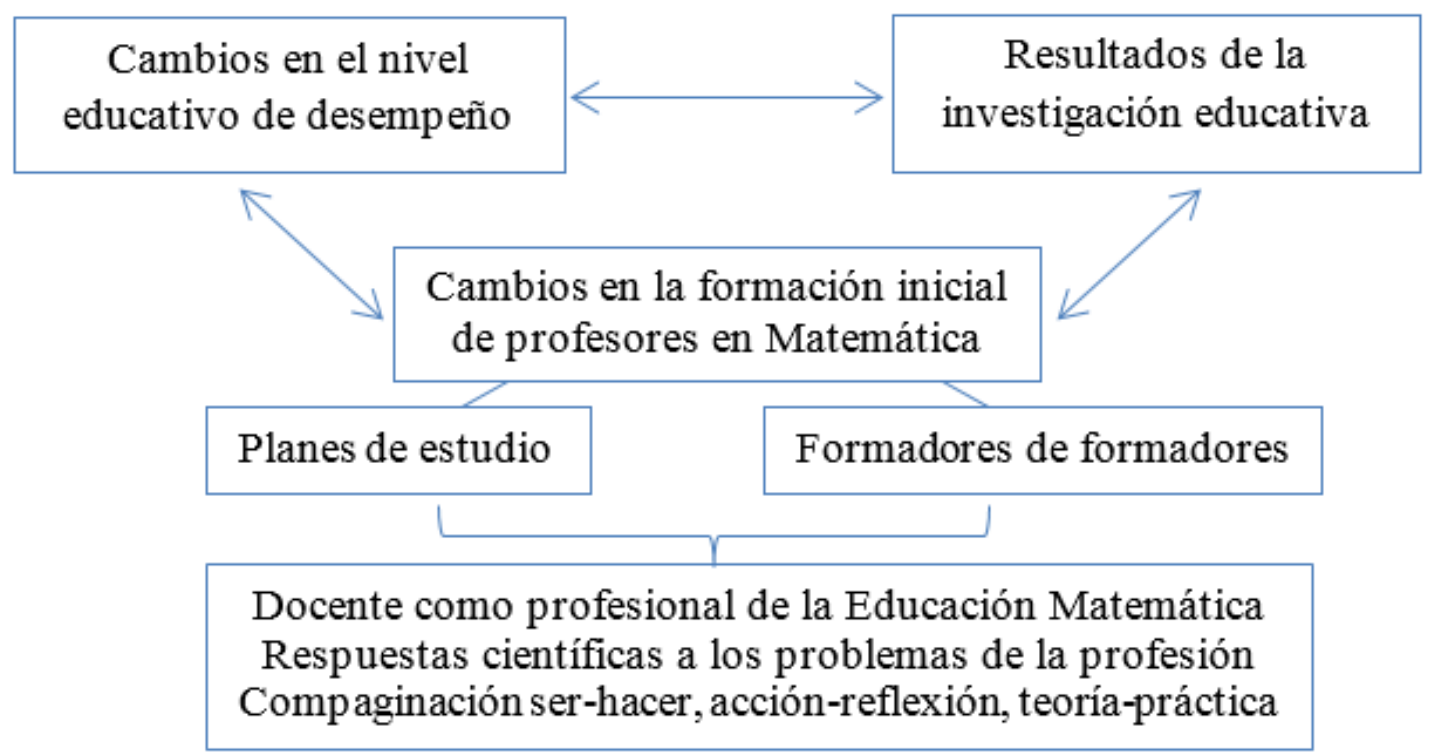

Fuente: Elaboración Propia

En la Fig. 2 se repasan los diversos componentes de formación inicial de profesores en Matemática desde los diseños curriculares, resumiendo a su vez algunas características y expectativas.

Figura 2. Componentes para la formación inicial de profesores en Matemática

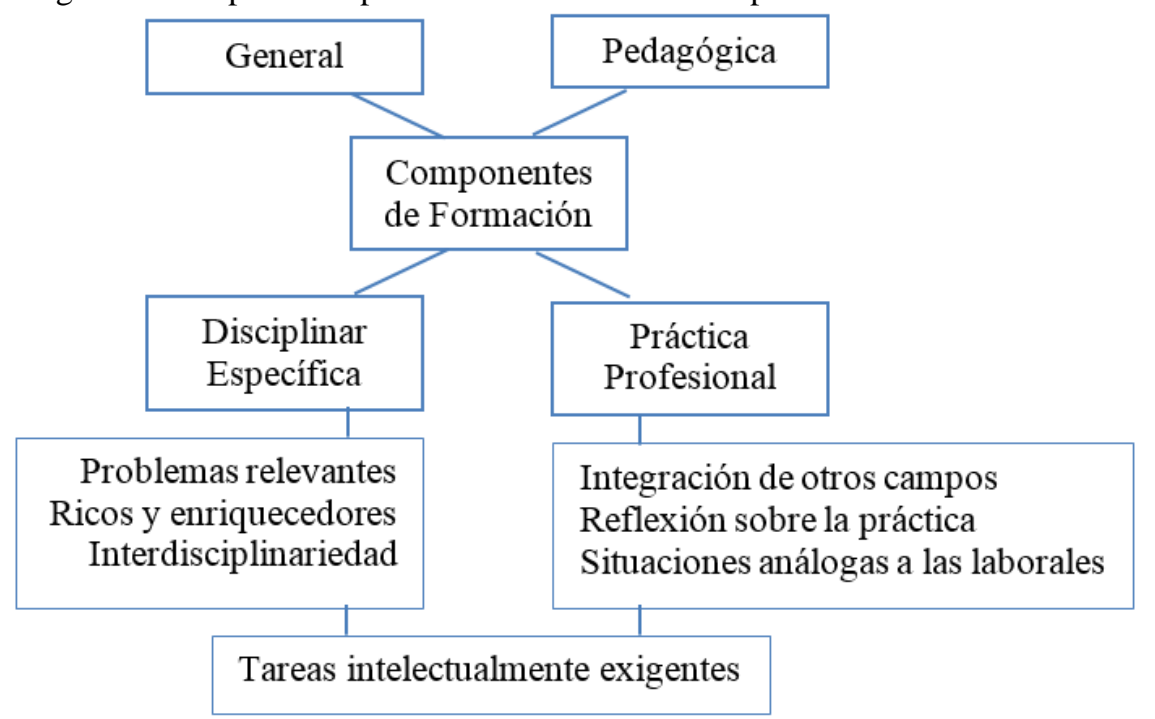

Fuente: Elaboración propia

En la Fig. 3 se procuran amalgamar los principales desafíos en estos tiempos identificados por los colegas que han publicado en Paradigma en relación con perspectivas para conceptualizar la formación inicial de profesores en Matemática. 
Figura 3. Desafíos para la práctica del profesor en Matemática en la actualidad

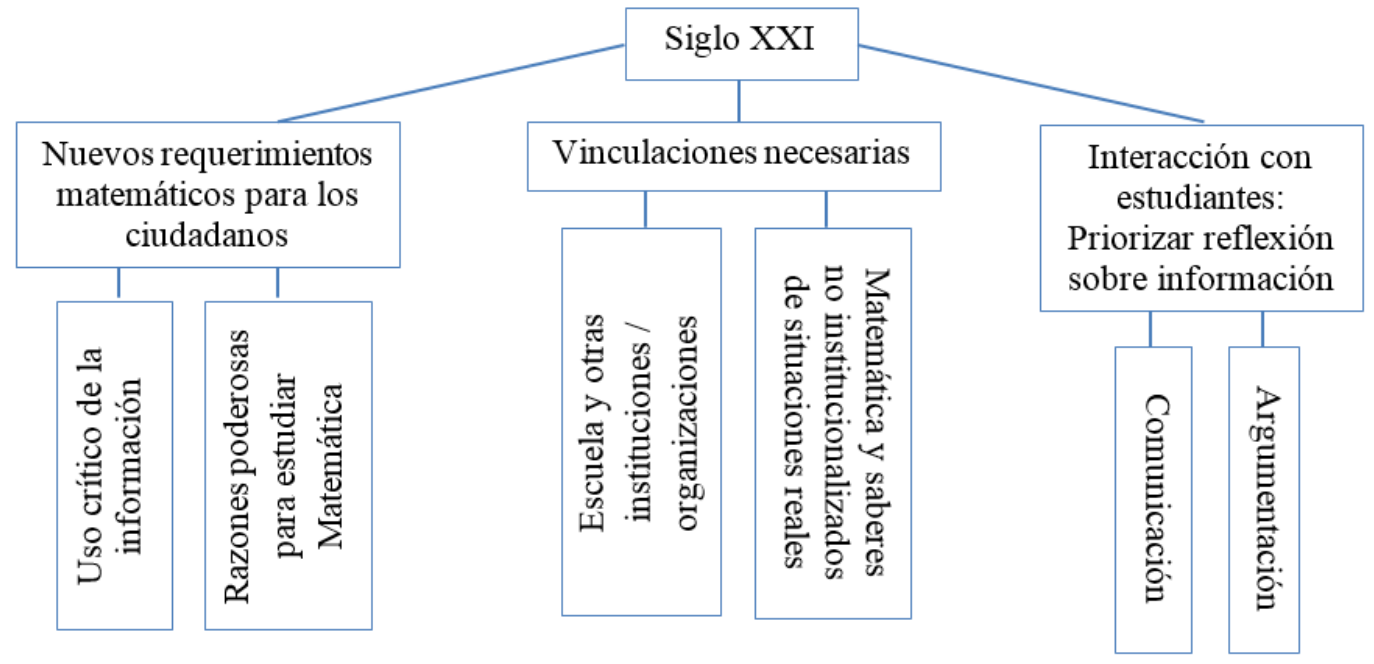

Fuente: Elaboración Propia

Dispositivos específicos de formación inicial de profesores en Matemática, con especial énfasis en las TIC

Las restantes seis publicaciones son de colegas de Brasil, Colombia, España y Venezuela. Tienen en común la puesta en valor de mecanismos que se consideran especialmente poderosos para empoderar a los futuros profesores en tareas de su labor que se consideran esenciales. Entre ellas va cobrando cada vez más presencia la integración de las TIC en los procesos de enseñanza-aprendizaje-evaluación de la Matemática.

Entre estos trabajos se encuentra el de Oliveira Groenwald y Moreno Ruiz (2006), de Brasil y España, quienes trabajan en pos a desarrollar en futuros profesores en Matemática habilidades para utilizar las TIC en su labor futura. Esto tanto en términos comunicacionales como didácticos específicos, empleando para ello mapas conceptuales y plataformas educativas.

Convocan a que en la formación inicial se brinden propuestas para realizar transposiciones didácticas que permitan transformar la Matemática que se estudia en la Universidad a una adecuada a las necesidades de la Enseñanza Básica donde trabajará el futuro profesor. Estas propuestas tendrán como foco reflexionar, evaluar y desarrollar la capacidad de un trabajo didáctico innovador de calidad, con el uso de las TIC atendiendo a la realidad de los estudiantes.

En sintonía con estas ideas, Richit y Maltempi (2009), de Brasil, parten considerando que la apropiación del conocimiento matemático requiere articularse con el uso didáctico de 
las TIC con el objetivo de favorecer las prácticas docentes de los futuros profesores. Analizan ambientes en los que el profesor en formación precisa motivar la investigación y reflexión en tanto mediador de la interacción que se da entre los estudiantes y las TIC en pos a la producción matemática.

Ven como una actividad favorable para ello el trabajo mediante proyectos y recogen testimonios de futuros profesores en Matemática que experimentaron este tipo de dispositivo. Entre las virtudes que sobresalen se encuentran el fomento de la autonomía, de la autoestima y del interés de los involucrados al poder estudiar temas que les gustan y que asumen con un valor intrínseco.

Apuntan que, mediante esta estrategia didáctica, los estudiantes desarrollan simultáneamente componentes de la formación específica, pedagógica y tecnológica, integrándolos desde la puesta en acción. También señalan que las habilidades de visualización de relaciones, experimentación, verificación de conjeturas y demostración de propiedades se ven altamente favorecidas.

En este sentido, recalcan la importancia de incorporar TIC en la formación de los profesores en Matemática, acompañadas de nociones de uso pedagógico de las tecnologías. Advierten que las comprensiones de los futuros profesores en Matemática acerca del papel de las TIC en la enseñanza están influenciadas por sus vivencias educativas. Se trata de concepciones que, a su vez, son dinámicas dado que se van modificando a medida que se amplían las experiencias al explorar nuevos modos de uso. De allí la relevancia que tiene trabajar con ellas en el período de formación inicial.

Puntualmente identifican seis etapas del proyecto llevado a cabo empleando TIC: elaborar un plan inicial, planificar la construcción de las representaciones, construir las representaciones respectivas, sistematizar las actividades elaboradas, evaluar lo desarrollado y socializar con la comunidad. Concluyen que este tipo de experiencias favorece la formación integral de los futuros profesores en Matemática.

También, Rojas Torres y Parra Sandoval (2009), de Venezuela, presentan elementos a tener en cuenta para la implementación de software educativo atendiendo a la comunicación y construcción del conocimiento didáctico matemático del profesor en Matemática en formación. Se preguntan, entonces, acerca de los tipos de experiencias formativas que estarían propiciando su construcción, de las maneras de integrarlas en las fases de planificación, 
gestión y evaluación que la tarea docente comprende y de las prioridades a focalizar para fomentar profesionales competentes, prácticos y coherentes.

Básicamente indagan en posibilidades de formación de profesores en Matemática para que aprendan a enseñar atendiendo a los procesos de aprendizaje. Entre los problemas advierten que muchas veces se produce desarticulación entre los distintos espacios de la carrera. Proponen, para mejorar el panorama, establecer canales a través de la didáctica específica.

También visionan en el diálogo en clase una posibilidad didáctica para dinamizar conjeturas y argumentaciones cuando se emplea software educativo, así como para situar al estudiante en diferentes contextos, resoluciones y contenidos. Se manifiestan a favor de aprovechar entre todos en las clases las diversas potencialidades del software y le otorgan importancia al discurso en la configuración del conocimiento didáctico matemático a través de formas de pensamiento que propicien un pensamiento crítico compuesto por razonamientos a favor y en contra de una cierta idea.

Finalmente expresan que una formación articulada en el sentido que se fue desarrollando contribuye a un educador actualizado a la altura de la Educación Matemática contemporánea, pudiendo integrar Didáctica, Matemática y Tecnología a partir de reflexionar en y sobre su práctica para poder ir transformándola.

Es en este sentido que León Gómez, Bara y Azocar (2013), de Venezuela, comentan que un profesor en Matemática requiere, además de conocimiento matemático a nivel superior, habilidades para planificar, gestionar y evaluar el contenido matemático. Pero, por lo general, resultan escasas las oportunidades que se brindan durante la formación inicial para aprender a enseñar Matemática desde la perspectiva de aprender una práctica. Para su análisis, se detienen en las categorías de conocimiento matemático escolar, conocimiento profesional y competencias profesionales del profesor de Matemática.

Con respecto al conocimiento matemático escolar, aclaran que este es un conocimiento proyectivo, en el sentido de que no es para su uso exclusivo, sino para hacerlo llegar a otros a través de la enseñanza. Advierten que muchas veces el profesor en Matemática, incluso con dominio disciplinar, presenta limitaciones en su comprensión cuando es puesto en situación de favorecer aprendizaje en un nivel básico en términos de Matemática escolar. Concluyen que dilucidar los significados de un concepto matemático es tarea fundamental en la planificación 
de la enseñanza y aprendizaje, constituyéndose en elemento clave de la formación del profesor en Matemática.

Acerca del conocimiento profesional, acuerdan con otros autores en que aglutina distintos tipos de conocimientos propios del profesor que lo ayudan a sostener enseñanzas en pos al aprendizaje de la Matemática y resaltan como esperable el análisis didáctico de un tema matemático dentro de la planificación.

Por su parte, las competencias profesionales del profesor en Matemática involucran posibilidad de realización de tareas tales como asumir proyectos de investigación en Educación Matemática, valorar los antecedentes históricos de la producción científica en Matemática y Educación Matemática, elaborar unidades didácticas específicas, crear nuevos escenarios para la enseñanza de la Matemática potenciados por una multiplicidad de entornos de la mano de las TIC, comunicar ampliamente resultados en clave de investigación en Educación Matemática, dominar con profundidad los saberes matemáticos, resolver problemas mediante estrategias cognitivas y metacognitivas.

Con base en otras investigaciones, consideran herramientas, como los mapas de enseñanza-aprendizaje y el análisis didáctico, para favorecer las planificaciones en términos de procesos no necesariamente lineales en manos de docentes reflexivos.

Específicamente, Carmona-Mesa y Villa-Ochoa (2017), de Colombia, identifican necesidades en la formación docente con respecto al uso de TIC, basándose en una revisión de estudios empíricos, y que puedan aportar a los procesos formativos del profesor en Matemática. Fue así que identificaron seis tipos de necesidades: experiencial, funcional, actitudinal, ética, crítica y pragmática. Además, proponen comprender su génesis y evolución. Como experiencias significativas para orientar procesos formativos de futuros profesores en el uso de TIC apuntan diseño, planificación y elaboración de lecciones, fortalecimiento de habilidades para manipular tecnologías educativas, implementación de diseños de clase en contextos reales, análisis de registro de prácticas, abordaje de elementos pedagógicos y didácticos para el uso de tecnología y evaluación de los procesos de formación.

En la necesidad funcional advirtieron que, por un lado, los profesores en formación poseen saberes tecnológicos previos pero no necesariamente de uso en el campo educativo. Por otro lado, encuentran que el factor edad también condiciona el desempeño con TIC, con 
mejores resultados en los más jóvenes. Por ello sugieren que formar en los aspectos técnicos no se restrinja solo al tramo de formación inicial de profesores en Matemática.

En cuanto a la necesidad de formación actitudinal, al indagar y transformar los modos de actuar al usar TIC, resaltan las creencias y confianza del futuro profesor. Identifican creencias de uso diversificadas, potentes y subversivas, que se corresponden con prácticas del tipo integrativas, incrementales, incidentales y hostiles, respectivamente.

Esto conlleva a una necesidad de formación ética profesional, en términos de una relación dialógica. Se presenta la necesidad de formar profesores reflexiones que se autoevalúen con respecto al uso de las TIC en su tarea docente y que sepan nutrirse colectivamente de conocimientos y experiencias a partir del trabajo colaborativo.

De este modo, la necesidad de formación crítica se refiere a poner en valor tanto potencialidades y fortalezas como debilidades en el uso de TIC en el tramo de formación inicial. Se requieren profesionales reflexivos en los contextos situados de desempeño laboral, que analicen con criterio la pertinencia y funcionalidad de las propuestas con TIC.

Esto se condice con una necesidad de formación pragmática, entendida como la proyección de la tarea laboral del ejercicio profesional mediante experiencias reales en instituciones educativas durante la carrera, previo a recibirse.

Luego del recorrido realizado, sugieren incluir propuestas transversales durante la carrera que integren el uso de TIC en los cursos específicos.

Por su parte, Oliveira Groenwald y Llinares (2019), de Brasil y España, ponen en valor el desarrollo en el futuro profesor en Matemática de la competencia observar con sentido, reconociendo que mientras más experiencias hayan vivido y más formados estén, más jugosa harán dicha observación. Entienden a esta competencia como la capacidad de acción del profesor en condiciones específicas, en el marco de una profesión compleja, esto es, en la que el profesional requiere ir renovándose periódicamente.

Particularmente, poder observar con sentido comprende la capacidad de identificar y comprender la situación que se esté observando, como por ejemplo producciones estudiantiles. Abarca las habilidades de identificar los aspectos relevantes de la situación, interpretar las interacciones en el aula dentro del contexto y tomar decisiones de acción. Precisamente implica que el profesor sea capaz de reconocer acontecimientos en el aula que puedan ser relevantes para explicar el aprendizaje de conceptos matemáticos. Esto es, además de 
conocimientos, requiere tener información adecuada para decidir con sensatez acerca de su enseñanza.

Advierten que esta competencia de observar con sentido se desarrolla gradualmente, provocándose su comienzo en la formación inicial y continuando durante toda la trayectoria profesional docente. En la misma resulta fundamental discernir cuál es la demanda cognitiva de la situación de enseñanza que, con base en otros autores, resumen en cuatro posibles niveles: de reproducción y memorización, de aplicación de información, de establecimiento de relaciones y de comprensión conceptual.

Finalmente indican que para desarrollar la competencia en cuestión sirve mucho analizar los registros de las prácticas, tanto propias como ajenas, durante la formación inicial del profesor en Matemática.

A continuación, mediante las Fig. 4 a 6, se procuran ilustrar esquemáticamente los aportes de las publicaciones consideradas con relación a dispositivos específicos de formación inicial de profesores en Matemática, con especial énfasis en las TIC.

De este modo, en la Fig. 4 se compendian los principales dispositivos indicados como especialmente favorables para una formación de calidad de los profesores en Matemática. En todos ellos se comparten experiencias llevadas a cabo en instituciones iberoamericanas y se reconoce que a través de los mismos se integran los diversos campos de formación de la carrera. No solo sirven en la instancia de formación inicial sino como modelos factibles a ser implementados luego en los niveles educativos de desempeño de los profesores en Matemática.

Figura 4. Ejemplos de dispositivos de formación especialmente potentes

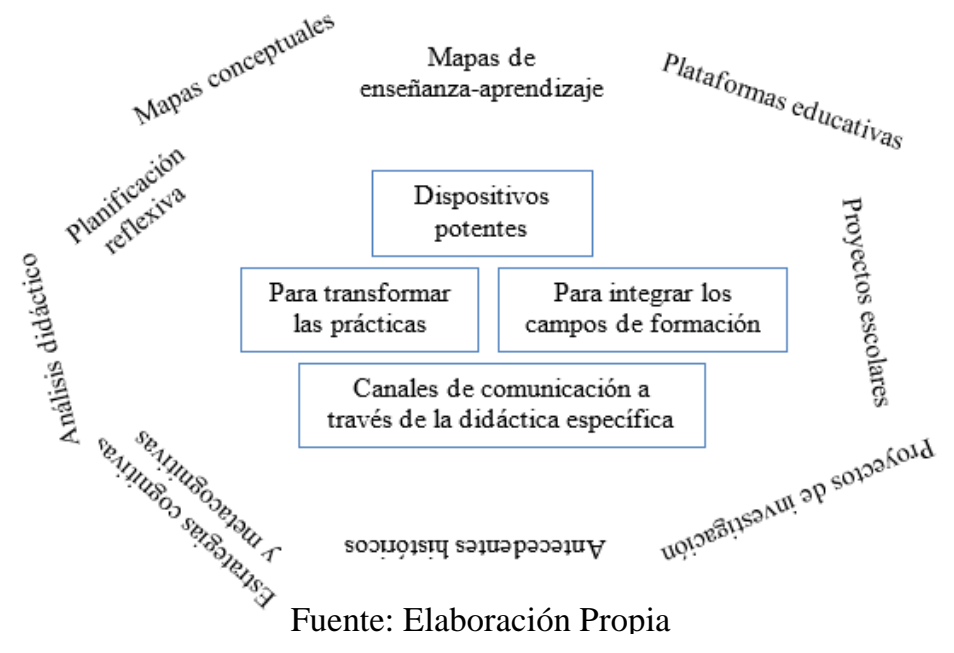


Específicamente en la Fig. 5 confluyen las condiciones que se han ido señalando para una incorporación consciente de las TIC al trabajo del futuro docente. Se subraya la vivencia en primera persona de experiencias formativas para que se pueda integrar con los conocimientos didáctico y matemático trascendiendo una faceta técnica o descontextualizada.

Figura 5. Requerimientos para la apropiación de las TIC en la formación del profesor en Matemática

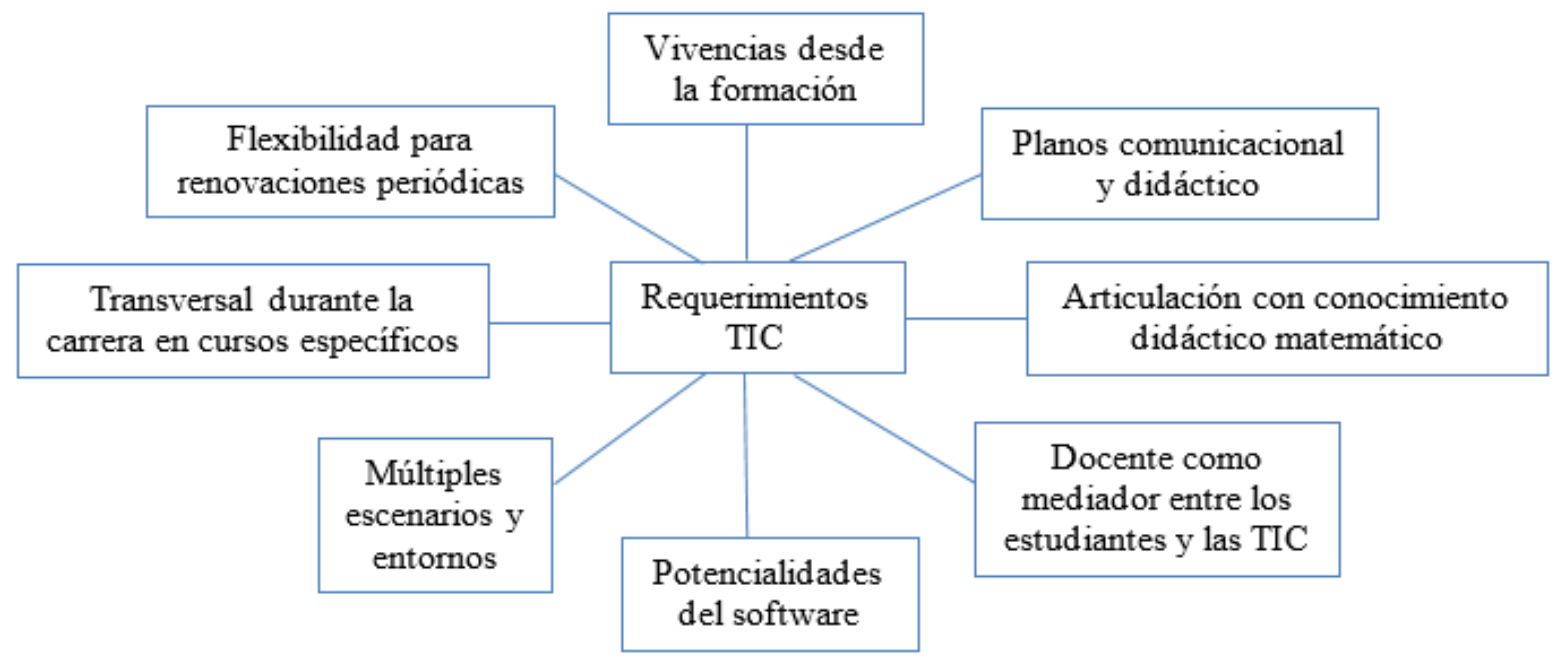

Fuente: Elaboración Propia

Todo ello debe ir acompañado de un diálogo en la clase (Fig. 6), a modo de interrogatorio didáctico, en el que docentes y estudiantes interactúan entre sí para favorecer el desarrollo de habilidades de pensamiento. Esto es, el dispositivo o recurso que se emplee dependerá del trabajo que los involucrados realicen para aprovecharlo en todo su potencial.

Figura 6. Posibilidades a desarrollar mediante el diálogo en clase que acompaña el uso de dispositivos

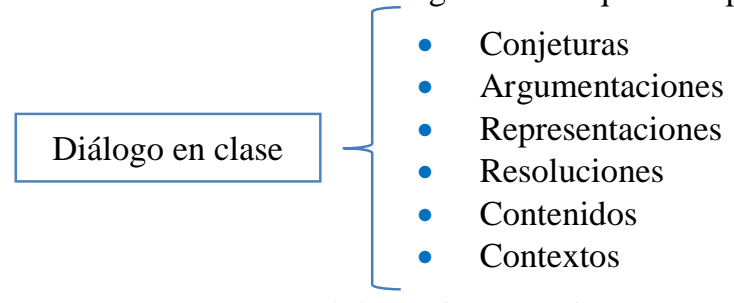

Fuente: Elaboración Propia

\section{A modo de cierre}

Actualmente me encuentro trabajando junto a un equipo de jóvenes investigadores en el Proyecto de Investigación: "El trayecto de la Práctica Profesional Docente en el Profesorado en Matemática. El caso de la Universidad Nacional de Rosario (Argentina)” así como en Plan de Posdoctoración "El campo de formación en la Práctica Profesional Docente en los 
Profesorados en Matemática de las Universidades Nacionales”, ambos de la Universidad Nacional de Rosario (Argentina).

Nos hemos abocado a analizar tanto los planes de estudio de las carreras en cuestión de nuestro país, Argentina, como los dispositivos de formación inicial de profesores en Matemática. También, hemos estudiado la configuración del conocimiento matemático para la enseñanza en ramas específicas y fuimos reconociendo, ya desde proyectos anteriores, marcas de docentes memorables en la biografía escolar de los futuros profesores en Matemática.

Nuestros hallazgos, en correlación con los aquí reportados, convocan a interpelar el aula de formación en los distintos planos del curriculum: planes de estudio, programas académicos y dispositivos de formación. Cómo nutrir las prácticas de los formadores de formadores con los resultados de la investigación educativa en Matemática, de qué modo propiciar la vivencia de las innovaciones didácticas en Matemática desde el aula de formación, mediante qué instrumentos articular convenientemente la evaluación en Matemática con los procesos de enseñanza y aprendizaje... son cuestiones que están en nuestra mira. Todo ello, a su vez, propiciando el foco de formación de profesionales que proyecten en sus estudiantes una ciudadanía plena, de personas críticas que se valgan convenientemente de la Matemática para desempeñarse en contextos altamente tecnologizados y cambiantes.

Por ello considero que sostener e ir mejorando estos espacios colectivos de puesta en valor de los trabajos investigativos de educadores no es una tarea automática. Me refiero a las Revistas así como a los Congresos, Posgrados, Redes, Proyectos... Esta sostenibilidad requiere convicción, colegialidad, decisión, esfuerzo, visión, compromiso... De allí mi especial reconocimiento a esta querida Revista Paradigma. ¡A celebrar!

\section{Referencias}

Carmona-Mesa, J.A. y Villa-Ochoa, J.A. (2017). Necesidades de Formación en Futuros Profesores para el Uso de Tecnologías. Resultados de un Estudio Documental. Paradigma, 38(1), 169-185. Recuperado de http://revistaparadigma.online/ojs/index.php/paradigma/article/view/606/603.

Fernández Peró, M., Hernández Murias, G., Sobrado Cárdenas, E. y Sampedro Ruiz, R. (2018). La Tarea Integradora desde un Enfoque Profesional Pedagógico en la Formación de Docentes. Paradigma, 39(1), 125-137. Recuperado de http://revistaparadigma.online/ojs/index.php/paradigma/article/view/646/643. 
Gomes de Oliveira, J.C. y da Silva, M.A. (2019). El Profesor de Matemática Idealizado por el Discurso de la Educación Matemática Crítica. Paradigma, 40(2), 31-51. Recuperado de http://revistaparadigma.online/ojs/index.php/paradigma/article/view/759/750.

González, F. (2000). Los Nuevos Roles del Profesor de Matemática. Retos de la Formación de Docentes para el Siglo XXI. Paradigma, 21(1), 139-172. Recuperado de http://revistaparadigma.online/ojs/index.php/paradigma/article/view/246/244.

León Gómez, N.A. (1999). La Formación del Docente de Matemática y la Reforma de la Educación Básica Venezolana. Paradigma, 20(2), 73-86. Recuperado de http://revistaparadigma.online/ojs/index.php/paradigma/article/view/235/233.

León Gómez, N., Bara, M. y Azocar, K. (2013). Planificación de la Matemática Escolar como Elemento Clave en la Formación del Docente. Paradigma, 34(2), 177-200. Recuperado de http://revistaparadigma.online/ojs/index.php/paradigma/article/view/524/521.

Oliveira Groenwald, C.L. y Llinares, S. (2019). Competencia Docente de Observar con Sentido Situaciones de Enseñanza. Paradigma, 40(1e), 29-46. Recuperado de http://revistaparadigma.online/ojs/index.php/paradigma/article/view/740/736.

Oliveira Groenwald, C.L. y Moreno Ruiz, L. (2006). Una Propuesta Metodológica para la Formación de Profesores de Matemáticas, utilizando Nuevas Tecnologías. Paradigma, 27(1), 209-223. Recuperado de http://revistaparadigma.online/ojs/index.php/paradigma/article/view/353/351.

Parra-Sandoval, H. y Villa-Ochoa, J.A. (2017). Vinculación de las Matemáticas con la Realidad. Implicaciones en la Conformación del Pensamiento Profesional del Docente. Paradigma, 38(1), 288-311. Recuperado de http://revistaparadigma.online/ojs/index.php/paradigma/article/view/611/608.

Pino-Fan, L.R. y Godino, J.D. (2015). Perspectiva Ampliada del Conocimiento DidácticoMatemático del Profesor. Paradigma, 36(1), 87-109. Recuperado de http://revistaparadigma.online/ojs/index.php/paradigma/article/view/552/549.

Richit, A. y Maltempi, M.V. (2009). Tecnologias Informáticas, Constructivismo y Enseñanza por Proyectos: Perspectivas de Formaçión Inicial para Profesores de Matemática. Paradigma, 30(1), 183-204. Recuperado de http://revistaparadigma.online/ojs/index.php/paradigma/article/view/431/428.

Rojas Torres, A. C. y Parra Sandoval, H. (2009). La Construcción del Conocimiento Didáctico Matemático al Utilizar Software Educativos. Paradigma, 30(1), 169-182. Recuperado de http://revistaparadigma.online/ojs/index.php/paradigma/article/view/430/427.

SHULMAN, L. S. (1986). Those Who Understand: Knowledge Growth in Teaching. Educational Researcher, 15(2), 4-14. Recuperado de: https://journals.sagepub.com/doi/10.3102/0013189X015002004\#articleCitationDownload Container https://doi.org/10.3102/0013189X015002004 